\title{
Symmetric Brownian motor
}

\author{
A. Gomez-Marin and J. M. Sancho \\ Departament d'Estructura i Constituents de la Materia, Facultat de Fisica, Universitat de Barcelona, Diagonal 647, \\ 08028 Barcelona, Spain
}

(Received 27 October 2004; published 8 February 2005)

\begin{abstract}
In this paper, we present a model of a symmetric Brownian motor which changes the sign of its velocity when the temperature gradient is inverted. The velocity, external work, and efficiency are studied as a function of the temperatures of the baths and other relevant parameters. The motor shows a current reversal when another parameter (a phase shift) is varied. Analytical predictions and results from numerical simulations are performed and agree very well. Generic properties of this type of motor are discussed.
\end{abstract}

DOI: 10.1103/PhysRevE.71.021101

PACS number(s): 05.40.-a, 05.70.Ln

\section{INTRODUCTION}

We all know that it is possible to extract some amount of mechanical work from a thermal bath at a temperature $T_{2}$ provided we have another bath at a lower temperature $T_{1}$ $<T_{2}$. Thermal engines are the devices that perform this task. All this is well known from elementary textbooks on thermodynamics. We also know from statistical mechanics that any object in a thermal bath exhibits random energy fluctuations of the order $k_{B} T$. These fluctuations are relatively very small for macroscopic objects but of very important relevance for nanometric objects such as biological motors: kinesins, dyneins, etc. [1]. We are also familiar with windmills, which are able to extract useful work from random winds by a proper adaptation to the wind direction. We can ask ourselves if it is possible to rectify thermal fluctuations by some appropriate mechanical devices.

The engines which aim to get useful work by rectifying thermal fluctuations are called Brownian motors (BM). In fact, the paradigm of such speculations is Feynman's famous ratchet and pawl machine [2]. During the last years, a lot of effort has been invested to study what has been called the ratchet effect. This is a mechanism which consists in breaking the spatial and temporal inversion symmetry of the system so that directed transport emerges, often enhanced by the thermal fluctuations. The ratchet mechanism can be implemented in different ways. Here we will make a model through an equation for a dynamical classical variable (position or angle) moving in a periodic and asymmetric potential (a ratchet potential) coupled with another degree of freedom which will break thermal equilibrium.

There is an enormous variety of ratchets in the literature: pulsating ratchets (on-off, fluctuating potential, traveling potential); tilting ratchets (fluctuating force, rocking ratchets), Seebeck ratchets, Feynman ratchets, temperature ratchets, frictional ratchets, quantum ratchets, collective ratchets, mechanico-chemical ratchets, ionic bombs and pumps, etc. [3].

In this work, we will focus on a new Brownian motor inspired by the so-called Feynman's ratchet (Fig. 1). Feynman stated in his famous lectures [2] that a very particular device (the ratchet and pawl machine) could have the efficiency of a Carnot engine when operating reversibly. He idealized a gadget (see Fig. 1) made up of two boxes with some gas at different temperatures. The hotter box contains an axle with vanes in it. The bombardments of gas molecules on the vane make the axle rotate with random symmetric fluctuations. At the other end of the axle there is a second box with a toothed wheel which in principle can turn only one way. The pawl (the stopping mechanism) is under the influence of another temperature (Fig. 2). At first glance one might think that it seems quite likely that the wheel will spin around one way and lift a weight even when both gases are at the same temperature, thus violating the Second Law. However, a closer look at the pawl reveals that it bounces and so the wheel will rotate randomly in any direction, doing a lot of jiggling and with no net turning. Thus, the machine cannot extract work from two baths at the same temperature.

When the temperature of the vanes is higher than the temperature of the wheel, Feynman concludes that some work is performed with Carnot's efficiency when the machine is lifting the weight very slowly. This is indeed a very optimistic result which has been revised, many years later, in Refs. 4 and 5. Indeed, there are some overlooked aspects in Feynman's argument which can be summarized as follows: since the engine seems to be simultaneously in contact with two baths at different temperatures (through the rigid axle), it cannot work in a reversible way and Carnot's efficiency will never be achieved. We will come back to this point later on in this paper.

A possible mathematical model of the device of Fig. 1 and Fig. 2 in terms of overdamped Langevin equations is

$$
\lambda_{1} \frac{d h}{d t}=-\partial_{h} V+\xi_{1}(t),
$$

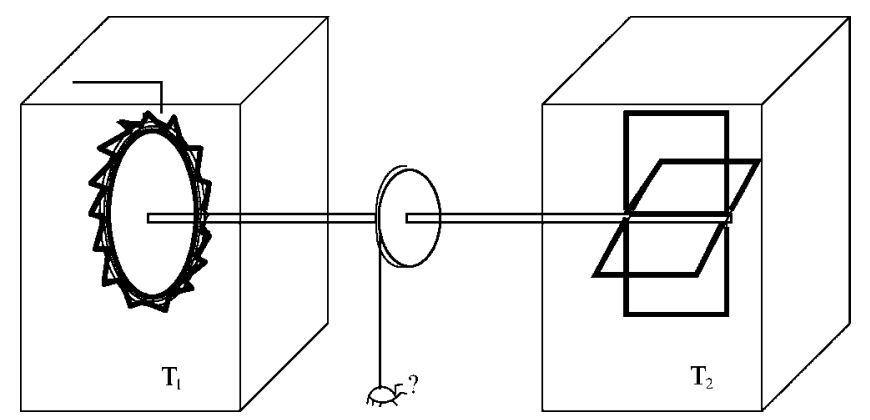

FIG. 1. Feynman's original ratchet and pawl machine. 


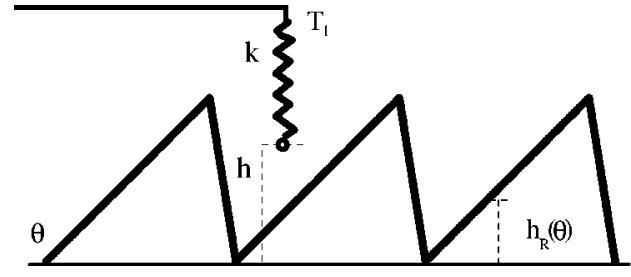

FIG. 2. Detail of the stopping mechanism based on the interaction between the ratchet and the pawl.

$$
\lambda_{2} \frac{d \theta}{d t}=-\partial_{\theta} V+\xi_{2}(t)
$$

where $\xi_{i}(t)$ mimics thermal fluctuations and it is assumed to be a white noise, Gaussian distributed with zero mean, satisfying the fluctuation-dissipation theorem,

$$
\left\langle\xi_{i}(t) \xi_{j}\left(t^{\prime}\right)\right\rangle=2 k_{B} T_{i} \lambda_{i} \delta\left(t-t^{\prime}\right) \delta_{i j} .
$$

The total potential energy is modeled by

$$
V(\theta, h)=\tau \theta+\frac{1}{2} k h^{2}+\frac{e}{e^{\left|h-h_{R}(\theta)\right| / l_{0}}-1},
$$

where $h_{R}(\theta)$ represents the periodic but asymmetric profile of the ratchet. An explicit expression will be given in the next section.

The potential $V$ has three terms. $\tau$ is the torque which gives useful work. The second term accounts for the potential energy stored in the spring (the pawl) with constant $k$, which pushes down the ratchet. Finally, the last term is a very repulsive potential at short distances and nearly zero at long ones. It is used to avoid the pawl crossing through the real physical surface of the ratchet. At the same time, it couples both degrees of freedom $\theta$ and $h$. Other potentials can account for the same physics in this mathematical scheme $[5,6]$.

Numerical simulations show a nonzero mean velocity of the ratchet device for very high temperature differences. The main conclusion is that, although the motor can perform useful work, it has an extremely small efficiency, being very far away from Carnot's efficiency estimated by Feynman, as pointed out and shown in Refs. [4-7]. This motor presents a high thermal conductivity and as a consequence has a very low efficiency.

The structure of this paper is the following. In Sec. II, we present the model for a symmetric Brownian motor and the numerical results obtained by computer simulations of the equations of such a device. Section III is devoted to the analytical approach to this model. We end in Sec. IV with some comments and conclusions.

\section{SYMMETRIC BROWNIAN MOTOR (SBM)}

Feynman's ratchet, and similar models, do not fulfill the following inversion property: $T_{2} \leftrightarrow T_{1} \rightarrow v \leftrightarrow-v$. Thus our initial motivation to propose this new model is to have a motor such that, when switching the temperatures of the two baths, that is, when reversing the temperature gradient, the mean velocity absolute value does not change while the sign

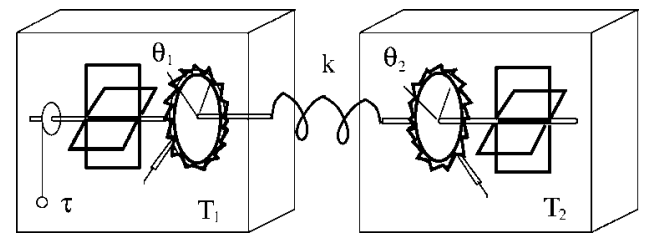

FIG. 3. Model for the symmetric Brownian motor (SBM).

of the velocity does. Obviously, this would imply some geometrical symmetries in the engine. One example of a microscopic model for a Brownian motor that suffers this change in the sign of the current can be found in Ref. [8]. There is also a model for a diode rectifier that is analytically solvable [9] and that has the same main features as the SBM, namely, it is symmetric and it shows "reversibility."

Our "symmetric" motor is shown in Fig. 3. According to this scheme, the stochastic differential equations that describe the dynamic evolution of the system in the overdamped regime are

$$
\begin{aligned}
& \lambda_{1} \frac{d \theta_{1}}{d t}=-\partial_{\theta_{1}} V+\xi_{1}(t), \\
& \lambda_{2} \frac{d \theta_{2}}{d t}=-\partial_{\theta_{2}} V+\xi_{2}(t) .
\end{aligned}
$$

The noise terms are again white thermal noises, Gaussian distributed and with zero mean, satisfying the fluctuationdissipation theorem (3).

The potential $V$ contains an external torque $\tau$, two ratchetshaped potentials, and a harmonic interaction that couples both degrees of freedom,

$$
V\left(\theta_{1}, \theta_{2}\right)=\tau \theta_{1}+V_{R}\left(\theta_{1}\right)+V_{R}\left(-\theta_{2}\right)+\frac{1}{2} k\left(\theta_{1}+\phi-\theta_{2}\right)^{2}
$$

where the phase shift $\phi$ is one of the most important parameters.

In Fig. 4, we see the form of the ratchet potential $V_{R}(\theta)$ used in the numerical simulations. Its analytical expression is

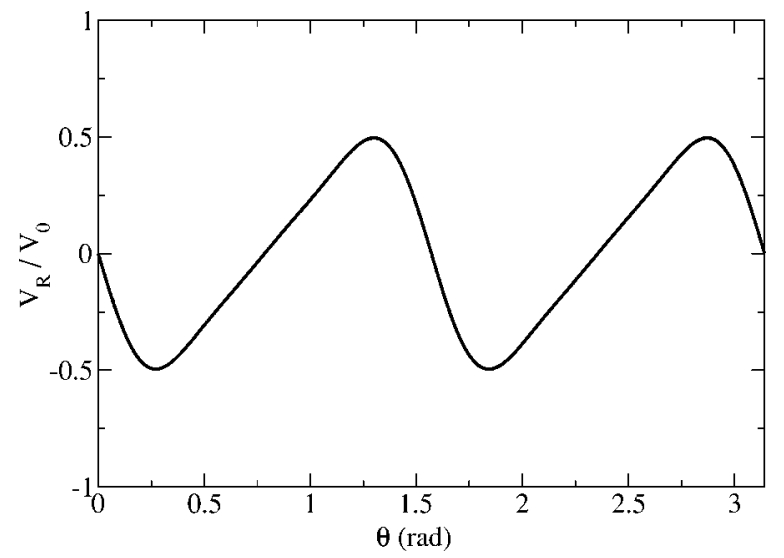

FIG. 4. Shape of the ratchet potential $V_{R}$ for $d=4$. 


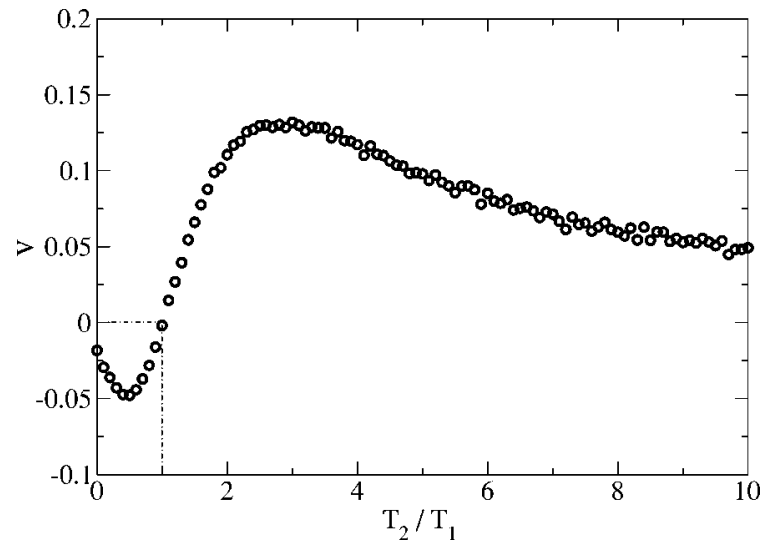

FIG. 5. Mean velocity versus $T_{2} / T_{1}(\tau=0)$.

$$
\begin{gathered}
V_{R}(\theta)=-\frac{V_{0}}{2.23} \mathcal{V}(\theta), \\
\mathcal{V}(\theta)=\sin (d \theta)+0.275 \sin (2 d \theta)+0.0533 \sin (3 d \theta) .
\end{gathered}
$$

$V_{0}$ controls the height of the potential, $d$ is the number of teeth per cycle $2 \pi$, and the asymmetry of the potential is controlled by changing the numerical coefficients that multiply the sinus functions expansion of the potential.

Notice that the ratchet potential that one variable $\left(\theta_{1}\right)$ sees is the specular image of the one that the other variable $\left(\theta_{2}\right)$ feels, as in the diode rectifier of Ref. [9]. It is in this sense that we call our model symmetric.

To study the relevance of each parameter, we proceed to express our system in terms of dimensionless ones. Introducing the dimensionless time $s$ as $t=\left(\lambda_{1} / V_{0}\right) s$, the set of Eqs. (5) becomes more compact. The dimensionless parameters are now $\widetilde{T}_{1}=k_{B} T_{1} / V_{0}, \widetilde{T}_{2}=k_{B} T_{2} / V_{0}, \tilde{k}=k / V_{0}, \tilde{\lambda}=\lambda_{1} / \lambda_{2}, \tilde{\tau}$ $=\tau / V_{0}$, and, of course, $\phi$, which is in radians. Notice that $V_{0}$ controls the energy scale, which is measured in $k_{B} T_{1}$ units. We also see, for instance, that only the fraction of the friction coefficients is relevant.

\section{A. Numerical results of SBM}

Preliminary numerical results indicate that the motor inverts its velocity when the temperature gradient is also inverted (see Fig. 5). Some important thermodynamic requirements are also fulfilled: there is no net motion in the limits $T_{2} \rightarrow T_{1}$ (a unique bath), $k \rightarrow 0$ (no coupling between baths), and $k \rightarrow \infty$ (only one effective temperature).

To perform the simulations we take $\tilde{\lambda}=1$ for simplicity and $k_{B} T_{1}=1$. As in previous models for motors, we have explored the parameter domain to get the most effective values (larger velocities). This situation corresponds to the parameter values $V_{0}=2.5, k=100$, and $d=16$. In Fig. 5 and Fig. 6 , some numerical results of the mean velocity $v \doteq\left\langle\dot{\theta}_{1}\right\rangle$ are presented for $\phi=0.4$. We see in Fig. 5 that there is a maximum around $T_{2} / T_{1}=2.5$ and a minimum at $T_{2} / T_{1}=0.5$. This implies that the velocity is bounded and larger temperature gradients do not imply larger velocities. For very large $T_{2}$, the motor does not see the ratchet potential and cannot take

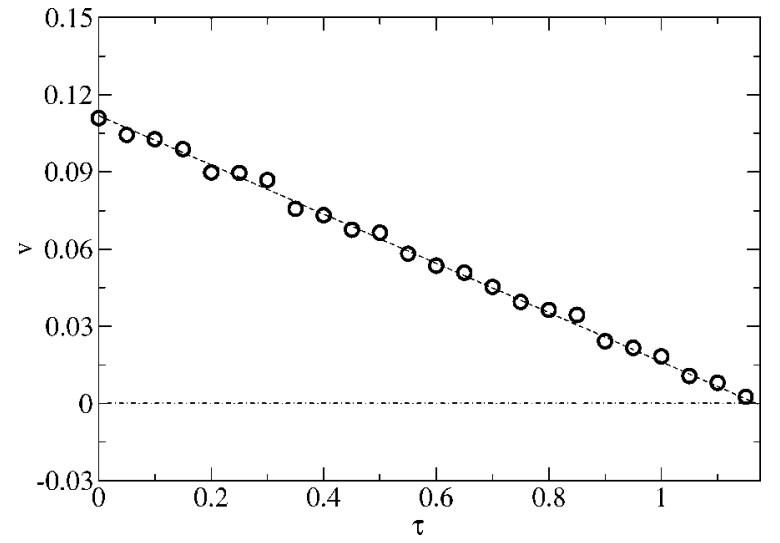

FIG. 6. Mean velocity of the motor at the optimal regime as a function of the external torque $\tau$. The stall torque (where $v=0$ ) is around $\tau=1.2$.

advantage of the broken inversion symmetry. Then the motor has a zero mean velocity. The linear dependence of the velocity around $T_{2} / T_{1} \sim 1$ is a clear signature that the motor has the inversion property. We will extend this result further. In Fig. 6, we see the expected negative linear dependence of the velocity versus torque until the stall force where the velocity is zero.

Moreover the most striking result is the velocity inversion as a function of the phase difference parameter $\phi$. We show in Fig. 7 the mean velocity of the motor as a function of the phase shift $\phi$ in the cases of $T_{2}=2 T_{1}$ and when $T_{1}=2 T_{2}$. One must notice that both cases are "symmetric." This result allows us to extract some conclusions, namely, the existence of a current inversion as a function of $\phi$, the great amplification of the speed for the particular value of $\phi=0.4$, and, finally, that the motor is perfectly symmetric when reversing the temperature gradient.

These results deserve theoretical explanations that will be presented in Sec. III.

\section{B. Energetics of the SBM: The efficiency}

It is not enough to know whether a motor will move, nor even how fast it will run. One would also like to know how

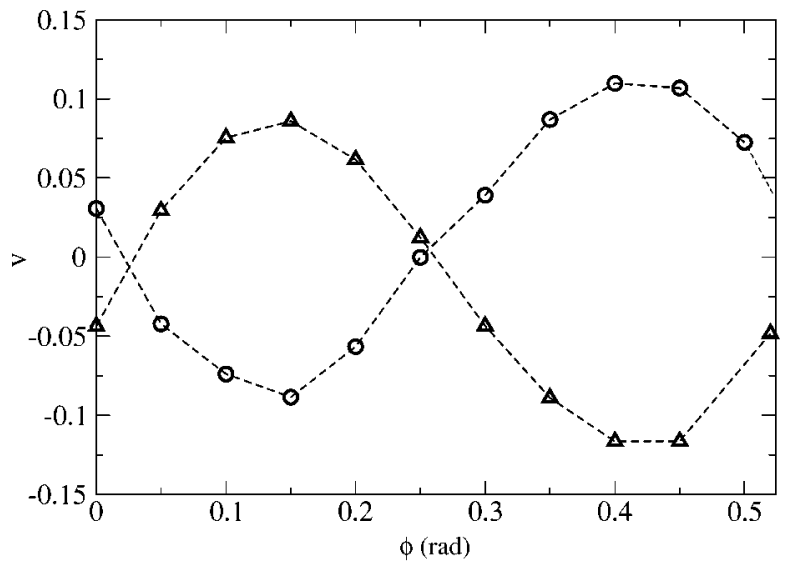

FIG. 7. Current inversion profile $v(\phi)$ when $T_{2}=2 T_{1}$ (circles) and when $T_{1}=2 T_{2}$ (triangles). The angle goes from 0 to 0.52 because we are considering $d=12$ and therefore the periodicity is $2 \pi / 12=0.52$. The dashed lines are a guide to the eyes. 
efficiently such a machine can operate. There are many different approaches in the literature to study energetics and, in particular, efficiencies $[6,10,11]$. We will follow Sekimoto's characterization of the energetics of thermal ratchet motors [6]. This scenario is the most suitable scheme both because it is intuitive and also from its potential applicability. The main idea is to find how much energy, received from an external source, the motor will be able to employ to produce useful mechanical work, i.e., to lift a load. In order to compute this, we must understand where the energy comes from, where it can go, and what ingredients account for that. Sekimoto deals with energies and not with power. When one tries to study the problem in units of power, velocities appear explicitly in the computations and, since they are instantaneously not continuous because of the stochasticity of the system, one finds that some of the terms behave extremely badly and it is impossible to get a reasonable numerical convergence in the simulations. Moreover, we have found it easier to directly compute the stochastic integrals and afterwards perform the average.

Let us analyze the energetics of such systems. We would expect there to be some energy $R$ that is released from the bath at $T_{2}$ (the one at a higher temperature) and that is partially converted into mechanical work $W$ to lift the load. However, according to the Second Law, some of this input energy must be dissipated $D$ into the heat bath at $T_{1}$. If the system has an intrinsic potential, there will also be a change in the potential energy denoted by $U$. So the law of the total energy conservation is

$$
R=D+W+U .
$$

Our interest here is to find the efficiency of the motor, which is defined, in general, as the ratio between the work extracted versus the energy input,

$$
\eta=\frac{\langle W\rangle}{\langle R\rangle} .
$$

The work $W$ is quite easy to calculate since it is just the torque times the angle displaced. Then the work performed during a time interval $t_{f}-t_{i}$ can be obtained as

$$
\langle W\rangle=\tau\left\langle\theta_{1}\left(t_{f}\right)-\theta_{1}\left(t_{i}\right)\right\rangle=\tau\langle v\rangle\left(t_{f}-t_{i}\right) .
$$

The evaluation of $\langle R\rangle$ needs a much more careful analysis. Following Ref. [6], we can write

$$
R=\int_{t_{i}}^{t_{f}}\left[\frac{\partial V\left(\theta_{1}(t), \theta_{2}(t)\right)}{\partial \theta_{2}}\right] d \theta_{2}(t) .
$$

So by using Eq. (6), we find that the energy that the bath at $T_{2}$ transfers to the mechanism in the bath at $T_{1}$ is

$$
R=\int_{t_{i}}^{t_{f}}\left\{-k\left[\theta_{1}(t)+\phi-\theta_{2}(t)\right]+V_{R}^{\prime}\left(-\theta_{2}(t)\right)\right\} d \theta_{2}(t) .
$$

We have to evaluate this integral for different realizations and perform usual statistical averages. We have observed that this quantity is always large, thus implying important losses of heat.

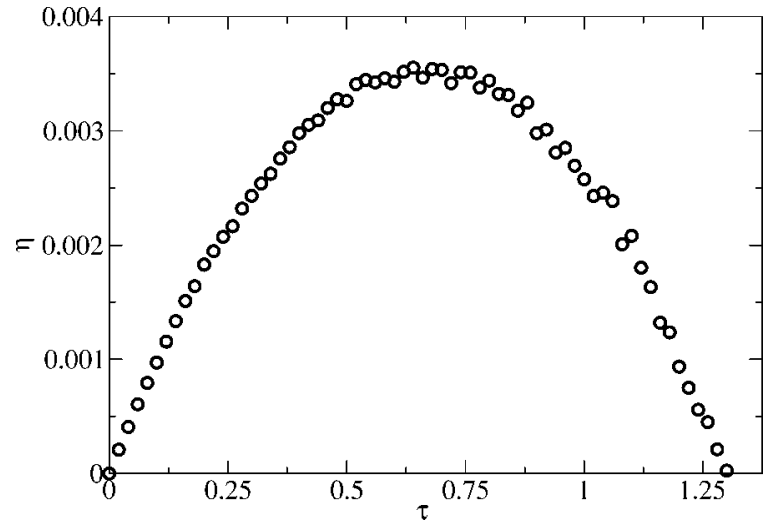

FIG. 8. Efficiency $\eta$ as a function of the torque $\tau$. The numerics are done at the optimal regime of the SBM.

To progress further, let us first note that the potential energy $U$ does not contribute because we have a cyclic engine. In fact, $V_{2}^{\prime}\left(-\theta_{2}(t)\right)$ is periodic and bounded and thus it can be discarded in the long time interval studied.

Thus the final expression for the efficiency that has to be evaluated numerically is

$$
\eta=\frac{\tau\left\langle\theta_{1}\left(t_{f}\right)-\theta_{1}\left(t_{i}\right)\right\rangle}{-k\left\langle\int_{t_{i}}^{t_{f}}\left[\theta_{1}(t)+\phi-\theta_{2}(t)\right] d \theta_{2}(t)\right\rangle} .
$$

In Fig. 8, the efficiency $\eta$ as a function of the torque $\tau$ is plotted. The specific values we use are around those that were shown to be the optimal ones; $V_{0}=3, T_{2}=2, k=100$, $\phi=0.4$, and $d=12$. The plot shows a parabolic curve for the efficiency with a maximum at the middle value of the stall torque. Notice that the maximum of the efficiency is still extremely low. Let us also underline that at the stall force $(\tau \simeq 1.25)$, the efficiency is zero, which means that unavoidable heat transfer occurs [4]. Therefore, even when $\langle v\rangle \rightarrow 0$, there is a nonzero heat flux transferred from the hotter bath to the cooler one. This is the reason why these motors have such low efficiencies.

\section{ANALYTICAL STUDY OF THE CURRENT INVERSION}

As we have seen in the numerical results, the $\phi$ parameter controls a spectacular phenomenon. The SBM exhibits velocity inversion when this parameter is varied. We know that this phenomenon exists and has been studied in the literature [12]. Our purpose now is to predict analytically that this happens in our model. Any kind of exact calculation in this system seems impossible and, so, some approximations have to be assumed.

Consider the equations that define our Brownian motor (5) written in the form

$$
\dot{\theta}_{1}=f\left(\theta_{1}\right)-k\left(\theta_{1}+\phi-\theta_{2}\right)+\xi_{1}(t),
$$




$$
\dot{\theta}_{2}=-f\left(\theta_{2}\right)+k\left(\theta_{1}+\phi-\theta_{2}\right)+\xi_{2}(t),
$$

where $f(\theta)$ is the force exerted by the ratchet potential and there is no torque. Let us define now the change of variables [13],

$$
\begin{gathered}
x=\frac{\theta_{1}+\theta_{2}}{2}, \\
y=\frac{\theta_{1}-\theta_{2}+\phi}{2} .
\end{gathered}
$$

The relevant variable $x$ describes the evolution of the center of mass and the "irrelevant" variable $y$ describes the relative motion of the two-particle system. Introducing a redefinition of the noises,

$$
\begin{aligned}
& \eta_{1}(t)=\frac{\xi_{1}(t)+\xi_{2}(t)}{2}, \\
& \eta_{2}(t)=\frac{\xi_{1}(t)-\xi_{2}(t)}{2},
\end{aligned}
$$

and using the fact that $y$ is very small, we can make a Taylor expansion up to first order, getting the pair of equations

$$
\begin{gathered}
\dot{x}=F(x)+y G(x)+\eta_{1}(t), \\
\dot{y}=Q(x)+y(R(x)-2 k)+\eta_{2}(t) .
\end{gathered}
$$

The explicit expressions for these quantities and further mathematical details are in the Appendix. Summarizing, we have found that the mean angular velocity $v$ can be expressed in the standard form $[12,14,15]$

$$
v_{\phi}=\frac{2 \pi}{d} N\left(1-e^{\beta_{\phi}}\right),
$$

where $N$ is a kind of normalization constant that does not depend very much on the parameter $\phi$. The relevant quantity $\beta$, which is a function of $\phi$, is given by

$$
\beta_{\phi}=\int_{0}^{L} d x \frac{-F(x)-\frac{Q(x) G(x)}{2 k}}{\frac{1}{4}\left[1+\frac{T_{2}}{T_{1}}+\left(1-\frac{T_{2}}{T_{1}}\right) \frac{G(x)}{k}+O\left(1 / k^{2}\right)\right]} .
$$

This expression can be numerically evaluated using the expressions for the different functions that appear in it. The value of the parameter $N$ has been estimated by using only one numerical value of the velocity obtained by the simulation (the minimum value at $\phi=0.25$ ). If $\beta$ changes its sign as a function of $\phi$, then the inversion phenomenon is predicted.

In Fig. 9, we compare the predicted theoretical dependence of the velocity as a function of the phase $\phi$ to the values obtained by numerical simulations of the model. The analytical result, considering the amount of approximations involved, traces very accurately the current inversion phenomenon. The fit is very encouraging and enlightening. The

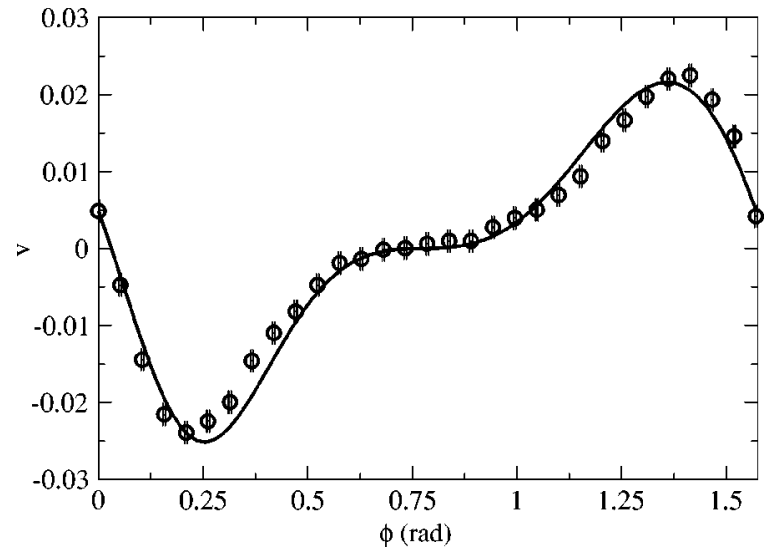

FIG. 9. Comparison of $v$ vs $\phi$ obtained from numerical simulations of the model (5) (circles) and from the analytical expression (23) (solid line). The parameters chosen are $d=4, k=100, V_{0}=3$, and $T_{2}=2 T_{1}$.

positions of the maxima are quite well determined, the inversion of current is clearly coincident with the simulations, and even the little shift near $\phi=0$ and $\phi=2 \pi / d$ is faithfully reproduced. This means that in our approximations we have kept the most dominant ingredients. Note that the $x$ axis goes from 0 to 1.57 because we have imposed $d=4(2 \pi / 4=1.57)$ in order to safely use the analytical expressions derived in the Appendix. However, for $d=12$ the computation of $\beta$ predicts qualitatively the current inversion too.

Now we will give an intuitive explanation of why the motor runs either forward or backward, depending on the value of the parameter $\phi$. The original problem can be mapped into a multiplicative noise scenario (see the Appendix for details) well documented in the literature [12,16-18]. Then we can straightforwardly identify in our case the effective potential $V_{\text {eff }}(x)$ [Eq. (A14)], which does not need to be asymmetric anymore, and the effective modulation $g_{\text {eff }}(x)$ [Eq. (A13)], which is responsible for the breaking of the symmetries that allow a nonzero mean velocity in the motor. What is more, by plotting them we can very intuitively see whether and why there is a forward or backward flux [16-18].

In Fig. 10, we plot, for three significative phase shifts, the noise coupling function $g_{\text {eff }}(x)$ and the effective potential $V_{\text {eff }}(x)$. A careful look at each of them easily reveals the current sign in a very intuitive way. The key point is the fact that when $g_{\text {eff }}(x)$ is large, the noise effects are amplified on one side of the hill of the periodic potential and, then, the net current will go in that direction. Then, it is much clearer that depending on the position of $g_{\text {eff }}(x)$ with respect to $V_{\text {eff }}(x)$ the current will go forwards, backwards, or, in the symmetric case, it will cancel. Thus, this simple scheme explains the qualitative behavior of the current inversion.

Two more comments should be made. Just for the sake of consistency, we have checked two basic conditions that must always hold on these kinds of motors. The first one concerns temperatures; if we set $T_{1}=T_{2}$, the integral $\beta_{\phi}$ vanishes because the denominator becomes constant and, therefore, the average velocity is zero. The second test is also related with the first one. When we make $k$ tend to infinity (this implies a 

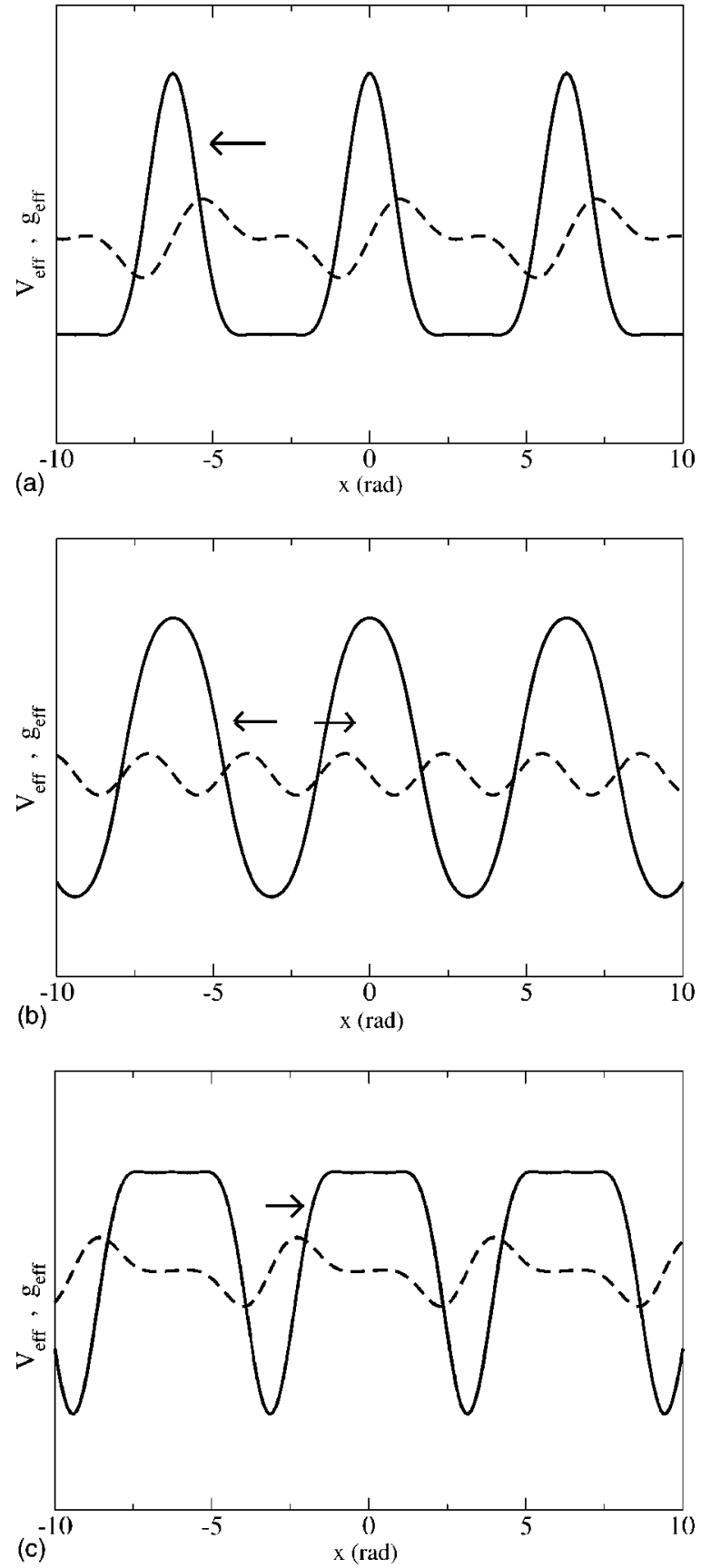

FIG. 10. Plots of the shape of the effective multiplicative noise $g_{\text {eff }}(x)$ (A13) (dashed line) and the effective potential $V_{\text {eff }}(x)$ (A14) (solid line) in arbitrary scales. The phase shifts are (from top to bottom) $\phi=0.25, \phi=0.785$, and $\phi=1.375$, which correspond to the fastest negative, zero, and fastest positive velocities respectively, for $d=4$. Arrows indicate the velocity direction.

rigid coupling and then only one effective temperature), the dependence on $x$ of the multiplicative noise disappears again (only additive noise is present) and, then, the motor cannot move anywhere on average.

\section{COMMENTS AND CONCLUSIONS}

We have presented and studied a symmetric Brownian motor. Its efficiency has been numerically obtained and other specific properties have been studied. This motor has a relevant external parameter (a phase) which induces the phenomenon of current inversion. We have performed an analytical calculation with appropriate approximations to get an expression for the mean velocity in terms of the relevant parameters of the model. This formal prediction fits very well with the data from the numerical simulations of the SBM model.

An important conclusion is that, even though the symmetric motor has a larger efficiency than other mechanical Brownian motors, the efficiency of such devices is very small. Regardless of the particular properties of these kinds of heat engines, they are anyhow unrealistic models for molecular motors since it is known that these biological systems do transform chemical energy into work, without the intermediate state of burning fuel. Consequently, one cannot think of these models as realistic ones for biological molecular motors.

Moreover, the mechanical coupling mechanism between both baths acts as a very good heat conductor even in situations of very small mean velocity. Therefore, the efficiency is only a small fraction of that of Carnot. This is in fact a general feature of heat Brownian motors due to the fact that in order to rectify thermal fluctuations, these systems must be put in contact and a lot of heat is interchanged. The diode rectifier of Ref. [9] does not present this problem and accordingly it can arrive near the Carnot efficiency in some limits.

\section{ACKNOWLEDGMENTS}

Fruitful discussions with Professor I. Sokolov, Professor J. M. R. Parrondo, and Professor C. Van den Broeck are gratefully acknowledged. This research was supported by the Ministerio de Educación y Ciencia (Spain) under project BFM2003-07850-C03-01.

\section{APPENDIX: ANALYTICAL APPROACH}

Our starting point is the pair of coupled equations (21) and (22) where the different functions are

$$
\begin{gathered}
F(x)=\frac{f(x-\phi / 2)-f(x+\phi / 2)}{2}, \\
G(x)=\frac{f^{\prime}(x-\phi / 2)+f^{\prime}(x+\phi / 2)}{2}, \\
Q(x)=\frac{f(x-\phi / 2)+f(x+\phi / 2)}{2}, \\
R(x)=\frac{f^{\prime}(x-\phi / 2)-f^{\prime}(x+\phi / 2)}{2} .
\end{gathered}
$$

Since the variable $y$ has a faster dynamics, we will first eliminate adiabatically Eq. (22), and we will also discard the function $R(x)$ because the parameter $k$ is very large. Then Eq. (22) reduces to 


$$
y=\frac{1}{2 k} \eta_{2}(t)+\frac{1}{2 k} Q(x) .
$$

Substituting now this expression in Eq. (21), we get a Langevin equation with two multiplicative noises,

$$
\dot{x}=H(x)+g_{1}(x) \xi_{1}(t)+g_{2}(x) \xi_{2}(t),
$$

where the new functions are

$$
\begin{gathered}
H(x)=F(x)+\frac{1}{2 k} Q(x) G(x), \\
g_{1}(x)=\frac{1}{2}\left(1+\frac{G(x)}{2 k}\right), \\
g_{2}(x)=\frac{1}{2}\left(1-\frac{G(x)}{2 k}\right) .
\end{gathered}
$$

Let us write now the Fokker-Planck equation associated to Eq. (A6),

$$
\partial_{t} P(x, t)=-\partial_{x} J(x, t)
$$

where

$$
\begin{aligned}
J(x, t)= & H(x) P(x, t)-K_{B} T_{1}\left[g_{1}(x) \partial_{x} g_{1}(x) P(x, t)\right] \\
& -K_{B} T_{2}\left[g_{2}(x) \partial_{x} g_{2}(x) P(x, t)\right] .
\end{aligned}
$$

After some manipulations with partial derivatives, the probability current above can be rewritten as

$$
J(x, t)=H(x) P(x, t)-\left[g_{\text {eff }}(x) \partial_{x} g_{e f f}(x) P(x, t)\right],
$$

where

$$
g_{\text {eff }}(x)=\sqrt{k_{B} T_{1} g_{1}^{2}(x)+k_{B} T_{2} g_{2}^{2}(x)},
$$

and $H(x)$ can be related to an effective potential,

$$
V_{e f f}(x)=-\int^{x} H\left(x^{\prime}\right) d x^{\prime} .
$$

Then we have to solve Eq. (A12) in the steady state for a constant flux $J$ with periodic conditions. The first step is to reduce this equation to a Bernoulli form which can be formally integrated. By imposing periodic boundary conditions, $P(x)=P(x+L)($ where $L=2 \pi / d)$, we get

$$
P_{0}\left(1-e^{\beta(L)}\right)=J \int_{0}^{L} d x \frac{e^{-\beta(x)}}{g_{\text {eff }}(x)},
$$

where $P_{0}$ is a constant that can be found by using the normalization condition $\int_{0}^{L} P(x) d x=1$, and $\beta$ is a relevant function whose expression is

$$
\beta(x)=\int_{0}^{x} d x^{\prime} \frac{-H\left(x^{\prime}\right)}{g_{1}^{2}\left(x^{\prime}\right)+\left(T_{2} / T_{1}\right) g_{2}^{2}\left(x^{\prime}\right)},
$$

where we take $k_{B} T_{1}=1$. Then the mean velocity $v \doteq\langle\dot{x}\rangle$ $\simeq\left\langle\dot{\theta}_{1}\right\rangle$ (since $\left.\left\langle\dot{\theta}_{1}\right\rangle \simeq\left\langle\dot{\theta}_{2}\right\rangle\right)$ is found to be proportional to $\left(1-e^{\beta(L)}\right)$. What is left to do is to find the $\beta$ integral for every $\phi$.

To simplify the calculation of the integral, we now make an expansion in powers of $1 / k$. Since the value of $k$ that makes the motor run faster is around $k=100$, one can safely suppose that the terms of the order $(1 / k)^{2}$ and so on will not notably contribute to the integral for $d<5$. Then, the integral (A16) turns out to be Eq. (24), which is evaluated numerically.
[1] G. Oster, Nature (London) 417, 25 (2002).

[2] R. P. Feynman, R. B. Leighton, and M. Sands, The Feynman Lectures on Physics (Addison Wesley, Reading, MA, 1963), Vol. 1, pp. 46.1-46.9.

[3] P. Reimann, Phys. Rep. 361, 57 (2002).

[4] J. M. R. Parrondo and P. Espanol, Am. J. Phys. 64, 1125 (1996).

[5] M. O. Magnasco and G. Stolovitzky, J. Stat. Phys. 93, 615 (1998).

[6] K. Sekimoto, J. Phys. Soc. Jpn. 66, 1234 (1997).

[7] T. Hondou and K. Sekimoto, Phys. Rev. E 62, 6021 (2000).

[8] C. Van den Broeck, R. Kawai, and P. Meurs, Phys. Rev. Lett. 93, 090601 (2004).

[9] I. M. Sokolov, Phys. Rev. E 60, 4946 (1999).

[10] D. Suzuki and T. Munakata, Phys. Rev. E 68, 021906 (2003).
[11] L. Machura, M. Kostur, P. Talkner, J. Luczka, F. Marchesoni, and P. Hanggi, "Brownian Motors: Current Fluctuations and Rectification Efficiency," e-print cond-mat/0409314.

[12] P. Reimann, Phys. Rep. 290, 149 (1997).

[13] S. Klumpp, A. Mielke, and C. Wald, Phys. Rev. E 63, 031914 (2001).

[14] H. Risken, The Fokker-Planck Equation (Springer, Berlin, 1984).

[15] B. Lindner, M. Kostur, and L. Schimansky-Geier, Fluct. Noise Lett. 1, 1 (2001).

[16] B. Lindner and L. Schimansky-Geier, Phys. Rev. Lett. 89, 230602 (2002).

[17] M. Buttiker, Z. Phys. B: Condens. Matter 68, 161 (1987).

[18] Ya. M. Blanter and M. Buttiker, Phys. Rev. Lett. 81, 4040 (1998). 